\title{
IMPACTO DE LAS PRÁCTICAS DE RECURSOS HUMANOS EN EL CAPITAL HUMANO Y LOS RESULTADOS ORGANIZATIVOS DE LAS EMPRESAS HOTELERAS
}

\author{
Jorge Plaza Bolaños* \\ Universidad de Las Palmas de Gran Canaria \\ https://orcid.org/0000-0003-2368-7266 \\ Julia Nieves* \\ Universidad de Las Palmas de Gran Canaria \\ https://orcid.org/0000-0001-9796-038X
}

\section{RESUMEN}

Existe un amplio consenso respecto a la conveniencia de mejorar la cualificación de los empleados en el ámbito de la hostelería y turismo. Asimismo, la literatura sugiere la importancia de las prácticas de RRHH en la mejora de los resultados organizativos. Este trabajo pretende aportar evidencia empírica del papel que las prácticas de reclutamiento-selección y de retención desempeñan como determinantes del capital humano y el desempeño organizativo en las empresas hoteleras. Los resultados muestran el importante papel que ejercen, fundamentalmente, las prácticas de reclutamiento-selección.

Palabras clave: Prácticas de RRHH de reclutamiento-selección; prácticas de RRHH de retención; capital humano; resultados organizativos

\section{The impact of human resources practices on human capital and the organizational performance of Spanish hotels}

\section{ABSTRACT}

There is an extended consensus related to the need to improve the qualification of workforce in tourism and hospitality industry. Likewise, the literature suggests the impor-

Fecha de recepción: 6 de mayo de 2019.

Fecha de aceptación. 4 de noviembre de 2019.

* Departamento de Economía y Dirección de Empresas. Facultad de Economía, Empresa y Turismo. Universidad de Las Palmas de Gran Canaria. Campus Universitario de Tafira. 35017. LAS PALMAS DE GRAN CANARIA (España).E-mail: jorge.plaza101@alu.ulpgc.es, julia.nieves@ulpgc.es 
tance of HR practices to improve organizational results. This paper aims to show empiric evidence about the role of recruitment-selection and retention practices on the human capital development and organizational performance in hotel firms. The results show the important role of recruitment-selection practices, particularly.

Keywords: Recruitment and selection HR practices; retention HR practices; human capital; organizational performance.

\section{INTRODUCCIÓN}

La literatura presenta consenso al señalar que las prácticas de recursos humanos (RRHH) contribuyen a desarrollar el carácter inimitable de los recursos de la empresa (Delery y Roumpi, 2017; Wright, Dunford y Snell, 2001). Por consiguiente, la gestión de las personas es una herramienta crítica para la competitividad de las empresas en general, y de las turísticas en particular (Frende-Vega, Biedma-Ferrer y Arana-Jiménez, 2017). Ello se traduce en la necesidad, tanto de la industria como de los teóricos, de examinar cómo afecta la gestión de RRHH al desempeño de las empresas (Madera et al., 2017).

Pueden considerarse dos perspectivas respecto a la gestión de RRHH. La primera propone la ventaja de los procesos organizacionales, que centra sus estudios en que las prácticas de alto rendimiento, cuando están alineadas a la estrategia empresarial y con la fuerza laboral, generan conocimientos, habilidades y destrezas, además de motivación y oportunidades que mejoran los resultados económicos (Becker y Huselid, 1998; Delery y Shaw, 2001). La segunda perspectiva considera que las prácticas de alto rendimiento son fácilmente imitables, por lo que no pueden constituir una ventaja competitiva sostenible (Wright, McMahan y McWilliams, 1994). Para este enfoque lo más relevante es la gestión del capital humano, el cual da la capacidad de proveer soluciones de gestión y atraer al personal más cualificado.

Para Delery y Roumpi (2017) ni las prácticas de alto rendimiento son tan fácilmente imitables porque, en ese caso, todas las empresas estarían usando actualmente las mismas, ni el capital humano es una ventaja sostenible dado que los trabajadores pueden abandonar la empresa en cualquier momento y llevarse el conocimiento a la competencia. Para estos autores, el mejor rendimiento se obtiene con una combinación de ambas.

Aunque la investigación sobre gestión estratégica de RRHH ha crecido en las últimas décadas, hay muy poca literatura sobre sus efectos en el contex to de la industria de la hostelería y el turismo (Madera et al., 2017). Este trabajo se centra en las prácticas de RRHH de reclutamiento-selección y de retención y trata de analizar sus efectos en el desarrollo del capital humano y en la consecución de resultados organizativos en las empresas de alojamiento turístico.

Investigaciones previas sugieren que las prácticas de RRHH para el reclutamiento, selección y retención favorecen el desarrollo del capital humano y la mejora de los resultados económicos en las empresas (Lepistö y Ihantola, 2018; Mahmood, 2015). No obstante, la relación de estos elementos ha sido escasamente estudiada en el ámbito de las 
empresas turísticas y, tampoco, se han identificado claramente las prácticas más efectivas para la obtención del mejor capital humano y los mejores resultados económicos (DíazFernández, López-Cabrales y Valle-Cabrera, 2014).

Por consiguiente, el propósito de este documento es el estudio de las prácticas de RRHH de reclutamiento-selección y de retención en empresas que gestionan establecimientos hoteleros en todo el territorio español. Se pretende evaluar el papel que desempeñan estas prácticas en el desarrollo de capital humano y en el incremento de los ingresos de explotación de las empresas. Las implicaciones de esta investigación se espera que puedan servir de base para la inversión y aplicación en diversas técnicas de reclutamiento, selección y retención del capital humano.

\section{REVISIÓN DE LA LITERATURA}

\subsection{Gestión estratégica de RRHH}

Los empleados representan un recurso clave para lograr los objetivos de las empresas porque sus conocimientos y comportamientos son esenciales para multitud de procesos organizativos (Allen, Ericksen y Collins, 2013). Particularmente, en el sector turístico, los RRHH constituyen uno de los principales activos que poseen las empresas (Kim et al., 2012; Kusluvanet al., 2010; Lillo-Bañuls, Ramón-Rodríguez y Sevilla-Jiménez, 2007). Por consiguiente, dichos recursos deben ser gestionados estratégicamente para alinear sus conocimientos, habilidades y conductas con los objetivos marcados por la organización.

Las empresas del sector de hostelería y turismo se enfrentan a un entorno altamente competitivo, donde la gestión de RRHH juega un papel clave en el éxito empresarial y, por tanto, en su subsistencia. Contrariamente a lo que sucede en otros sectores industriales, donde se ofrecen productos físicos o tecnológicos, en el ámbito de la hostelería y turismo el producto es intangible (e.g., experiencias, trato ofrecido), y la valoración del servicio por parte del cliente depende, en gran medida, de la interacción con los empleados de primera línea(Gutiérrez-Broncano y Rubio-Andrés, 2009; Madera et al., 2017). Por consiguiente, en este sector, los RRHH son los que condicionan la calidad del producto ofrecido, determinan cómo es percibida la empresa, y, además, ostentan un importante papel en la fidelización del cliente (Lillo-Bañuls, 2009), todo lo cual tiene un impacto directo en los resultados económicos.

En el campo de la gestión estratégica de RRHH relacionado con hostelería y turismo la literatura ha evolucionado en las últimas décadas y ha permitido aplicar sistemas de gestión que han proporcionado ventajas competitivas a las empresas. A pesar de la proliferación de estudios, se carece de información fiable sobre cómo las prácticas de RRHH impactan en las actuaciones de las empresas. La gestión estratégica de RRHH se puede considerar como el nexo entre las actuaciones del departamento de RRHH y el desempeño empresarial. En tal sentido, Kaufman (2012) sostiene que la gestión de RRHH es una inversión en la producción empresarial generalmente visto sólo como un gasto, pero que repercute positivamente en la empresa con un alto retorno económico.

Como señalamos anteriormente, este trabajo se centra en las prácticas de RRHH de reclutamiento-selección y en las prácticas de retención. Entendemos que son prácticas 
clave para el sector que se analiza debido a diversas razonas. Por una parte, porque la industria hotelera se caracteriza por la baja cualificación de su personal (Hjalager, 2002; Walsh, Enz y Canina, 2008) y la aplicación de prácticas que impliquen un proceso de reclutamiento riguroso, además, de valorar aspectos tales como el potencial, las habilidades para relacionarse o para trabajar en equipo de los candidatos, pueden constituir importantes herramientas para mejorar el nivel de capital humano de estas empresas. Por otro lado, la literatura reconoce que el sector,también, se caracteriza por un alto ratio de rotación de los trabajadores (Hjalager, 2002; Lam, Lo y Chan, 2002; Yang y Wan, 2004) lo que implica que la mayoría de los empleados carecen de experiencia y habilidades específicas, perjudicando, por tanto, el desempeño de las empresas.

\subsection{Prácticas de RRHH de reclutamiento y selección}

El reclutamiento es el proceso por el cual se obtiene un grupo de candidatos con las capacidades necesarias para optar por un puesto de trabajo en la empresa. Los procesos de reclutamiento no suelen ser una prioridad cuando se quiere cubrir la vacante con rapidez o cuando suponen un coste que no se quiere asumir, lo que provoca que la obtención de candidatos se vea afectada tanto en cantidad como en calidad. En cambio, las empresas que quieren captar a los mejores candidatos del mercado, realizan grandes esfuerzos para el correcto reclutamiento, sobre todo cuando se trata de puestos relevantes o críticos para el funcionamiento de la organización (Chan y Kuok, 2011).

Por su parte, la selección de personal es el proceso de elección de aquellos candidatos, de entre los reclutados, que se adecuen mejor al perfil del puesto de trabajo para el que están siendo considerados. La captación de las personas adecuadas para el puesto implica que la organización pueda delegar en el profesionalismo de los miembros del grupo y en su capacidad para compartir los valores comunes. Por ello, el correcto reclutamiento y posterior selección de personal contribuye a lograr los objetivos de la empresa (Lepistö y Ihantola, 2018).

Investigaciones previas han analizado la ventaja competitiva que supone disponer de personal mejor cualificado que la competencia (Lepistö y Ihantola, 2018; Mahmood, 2015). Existe un consenso generalizado en señalar que es vital la adquisición y el desarrollo del capital humano(Hamilton y Davison, 2018). No obstante, obtener este capital humano es una labor compleja y en la que influyen muchos factores, puesto que no solo se trata de seleccionar a los trabajadores con mejores habilidades y conocimientos, sino que, además, es necesario conseguir que encajen en la cultura organizacional y en el sistema de producción de la empresa.

\subsection{Prácticas de RRHH de Retención}

Las prácticas de RRHH de retención son aquellas que fomentan la permanencia en la empresa de los empleados. De este modo, si las relaciones laborales se mantienen en el tiempo, permiten a los individuos acumular conocimiento y habilidades específicas del sector, beneficiando, así, a las empresas que son capaces de retener a sus empleados. La retención ha sido definida como las fuerzas cognitivas, emocionales y comportamentales que un empleado enfoca de manera positiva para la consecución de los objetivos empresa- 
riales (Shuck y Wollard, 2010). Como consecuencia de ello, se entiende que las prácticas de RRHH enfocadas a lograr que esas fuerzas se presenten lograrían mejorar su desempeño. Investigaciones previas han mostrado que las empresas que tienen la retención del personal correctamente integrada en la gestión de RRHH obtienen una ventaja competitiva sostenible (Albrechtet al., 2015).

La retención del personal es fundamental para el desarrollo de la empresa. Para ello es necesario lograr la adecuada motivación del empleado, así como proporcionarle los recursos necesarios para que pueda desempeñar su labor de la manera más satisfactoria posible. Estudios previos indican que una correcta retención de personal provoca más compromiso, motivación y empoderamiento de los trabajadores. Como resultado, los empleados son menos absentistas y sus intenciones de dejar la empresa se reducen, además de aumentar su rendimiento y ofrecer comportamientos extra-rol, esto es, más allá de las responsabilidades atribuidas. Todo ello genera un cúmulo de beneficios para la organización que, tradicionalmente, ha sido poco valorado. Actualmente, tanto teóricos como profesionales sugieren que la implementación de correctas prácticas de retención proporcionan importantes ventajas a las empresas (Zhong, Wayne y Liden, 2016).

\subsection{Capital Humano}

Becker (2002) define el capital humano como el conjunto de conocimientos, habilidades, capacidades y creatividad del individuo. En la misma línea, para Pasban y Nojedeh (2016), las características del capital humano son la creatividad, innovación, conocimiento, habilidades, valor añadido, ventaja competitiva y mejora de la satisfacción del cliente.

El capital humano es uno de los recursos más difíciles de lograr, analizar y controlar, pero, a su vez, es el que marca la diferencia, sobre todo, en los sectores más influenciados por los RRHH como la hostelería y turismo. Muchos tipos de capital son considerados inputs para la producción de bienes y servicios, sin embargo, el capital humano no es simplemente un input ya que su rol está indefinido. El capital humano muestra un talento intrínseco que puede tanto cambiar o moldearse a sí mismo como alterar otros inputs (Pasban y Nojedeh, 2016). Así, el capital humano se considera valioso porque contribuye a la mejora de la eficiencia en la organización, colaborando en la reducción de costes o proveyendo beneficios adicionales a los clientes. Particularmente, el capital humano genera ventajas cuando es escaso o está especializado en las actividades específicas de la empresa. Si ambas situaciones se dan, se considera que el capital humano contribuye a la competitividad de la empresa(Díaz-Fernández et al., 2014).

\section{DESARROLLO DE HIPÓTESIS}

\subsection{Prácticas de reclutamiento-selección y capital humano}

Si el mercado de trabajo fuera perfectamente competitivo, de modo que los RRHH fueran homogéneos y dispusieran de total libertad de movilidad, los niveles salariales serían la clave para atraer, retener y reemplazar al personal de una organización. En esta situación, la inversión en políticas de RRHH para disponer del mejor capital humano no 
tendría su éxito garantizado. El aumento del coste en obtener mejor capital humano provocaría que el retorno económico fuera menor (Steffy y Maurer, 1988). En cambio, como el mercado ni es homogéneo, ni la movilidad es sencilla, para obtener los mejores RRHH no solo es necesario una inversión económica sino, además, plantear una estrategia acorde a los objetivos empresariales que se establezcan.

Según las definiciones de capital humano anteriormente descritas, en resumen, se considera como tal al conjunto conocimientos, habilidades, capacidades y destrezas que poseen las personas que constituyen una organización y que permiten el correcto funcionamiento de la misma. Entre las funciones del departamento de RRHH figuran la selección, socialización, formación, y pago por capacidades, lo que constituye una inversión en el capital humano específico de la empresa que puede favorecer la obtención de ventajas competitivas. Por tanto, los mecanismos aplicados por las empresas para establecer sus prácticas de RRHH deben ser rigurosos. Un sistema de RRHH mal enfocado puede dar lugar al reclutamiento y selección de individuos que no poseen el conocimiento, habilidades y capacidades especificas necesarias para la empresa o que tengan valores o actitudes incompatibles con los de la organización (Lado y Wilson, 1994).

En este estudio se propone que las empresas pueden aumentar su nivel de capital humano mediante un intenso proceso que incluya aspectos como numerosas entrevistas, pruebas, diferentes fuentes de reclutamiento, así como una rigurosa evaluación que permita seleccionar a los candidatos con un alto potencial y habilidades para relacionarse y trabajar en equipo. Considerando los anteriores argumentos, formulamos la primera hipótesis de trabajo:

H1. La adopción de prácticas de RRHH de reclutamiento-selección se relaciona positivamente con el nivel de capital humano de la empresa.

\subsection{Prácticas de retención y capital humano}

Captar y desarrollar el capital humano es importante para lograr los objetivos empresariales, pero,igual de importante es tratar de retenerlos en la organización. Los profesionales mejor capacitados habitualmente tienen la necesidad de crecimiento profesional y, si no son debidamente reconocidos en su propia empresa, tanto económica como profesionalmente, buscarán en la competencia esas oportunidades. Del mismo modo, para la competencia puede ser vital la adquisición de personal relevante de otras empresas y tratarán de captarlos ofreciéndoles mejoras en sus condiciones laborales actuales. Además, no sólo importa la pérdida decapital humano, sino, también, lograr su mejor rendimiento, ya que ciertos empleados que no se ven correctamente valorados, o sienten que la empresa no es justa con su desempeño, verán reducido su compromiso y con ello su rendimiento. Consecuentemente, es necesario que las empresas rentabilicen la inversión en captación y desarrollo de su capital humano con prácticas de retención enfocadas a mejorar la satisfacción laboral, el desarrollo de planes de carrera y mejoras sociales y económicas con el objetivo de reducir la rotación del personal y aumentar el compromiso.

Cuando las relaciones laborales se mantienen en el tiempo, permiten a los empleados adquirir experiencia, capacidades y habilidades propias de la organización, que constituyen componentes del capital humano. De este modo, mediante prácticas de retención, las 
empresas podrán beneficiarse de niveles más altos de este recurso (Leana y Van Buren, 1999). Por tanto, se plantea que las prácticas de RRHH de retención, al favorecer una baja rotación entre los empleados, pueden mejorar el nivel de capital humano en las empresas. Por consiguiente, se propone la siguiente hipótesis deinvestigación:

H2: La adopción de prácticas de RRHH de retención se relaciona positivamente con el nivel de capital humano de la empresa.

\subsection{Prácticas de reclutamiento-selección y resultados organizativos}

Investigaciones previas han mostrado que las prácticas de RRHH de reclutamiento y selección favorecen los resultados organizativos. Así, Naveen (2013) sostiene que implementar prácticas de reclutamiento y selección exitosas es clave para mejorar el desempeño económico. También, Mustapha, Ilesanmi y Aremu (2013) y Saifalislam, Osman y AlQudah (2014)muestran que existe una clara relación entre las prácticas de reclutamiento y selección y los resultados empresariales. Por su parte, Petts (1997) afirma que invertir en desarrollo de sistemas de selección válidos es fundamental puesto que disponer del personal adecuado puede mejorar y mantener el desempeño organizacional.

El éxito de la empresa en su sector depende del buen hacer de sus trabajadores. Estudios previos muestran que una insuficiente o inadecuada gestión de los trabajadores provoca mayores tasas de rotación y menor productividad y es una importante causa de fracaso empresarial. Por tanto, disponer de sistemas de RRHH efectivos ayuda a la organización a atraer y contratar a los mejores candidatos, los cuales pueden lograr mejoras cuantitativas y cualitativas para la consecución de los objetivos empresariales (Saddam y Mansor, 2015).

En síntesis, los RRHH son la clave de todas las operaciones de una organización ya que a cualquier nivel hay personal del que depende el desempeño, desde el mantenimiento técnico hasta la toma de decisiones. Por tanto, la selección efectiva de los RRHH es vital para la consecución de los objetivos empresariales. Por ello, es esencial que el departamento de RRHH sea capaz de seleccionar a los trabajadores adecuados para los puestos apropiados (Harky, 2018).

Atendiendo a lo anterior, proponemos que cuanto más efectiva es la organización reclutando y seleccionando trabajadores, más fácil será alcanzar resultados organizativos, particularmente mejorar sus ingresos de explotación. Planteamos, por tanto, la siguiente hipótesis de investigación:

H3. La adopción de prácticas de RRHH de reclutamiento-selección se relaciona positivamente con el incremento de los ingresos de explotación de las empresas.

\subsection{Prácticas de RRHH de retención y resultados organizativos}

La literatura señala que una de las claves para retener a los empleados es contar con personal comprometido con la organización. Las empresas con empleados comprometidos tienen mejores resultados de retención como consecuencia de la baja rotación del personal, mejores niveles de productividad, rentabilidad, y satisfacción del cliente, y la reducción de 
los niveles de absentismo laboral (Markos y Sridevi, 2010). Por consiguiente, la organización debe tener como objetivo desarrollar y nutrir el compromiso, el cual depende tanto de la empresa como del trabajador (Robinson, Perryman y Hayday, 2004).

Algunas prácticas de RRHH pueden fomentar la permanencia en la empresa de los empleados y, de este modo, retener un gran porcentaje de su capital humano (Minbaeva, Foss y Snell, 2009; Subramaniam y Youndt, 2005). Collins (2000), señala que ciertas prácticas de socialización permiten construir identidad con la organización y, por tanto, pueden reducir el nivel de rotación de los empleados. Para este autor, también, el nivel de remuneración salarial es uno de los principales factores que influyen en la decisión de los empleados de permanecer en la empresa o intentar la búsqueda de nuevas oportunidades. En general, las prácticas de retención implican inversiones de la empresa para aumentar el coste de abandonar la organización por parte de los empleados. La retención de los empleados es particularmente significativa en las empresas de alojamiento turístico debido a la importante interacción que se establece entre el personal de la organización y los clientes (Gutiérrez-Broncano y Rubio-Andrés, 2009). Se trata de un sector en el que la prestación del servicio presenta un alto grado de personalización o adaptación al cliente y los empleados con mayor experiencia y conocimiento pueden desempeñarlo con mayor eficiencia. En tal sentido, Walsh et al. (2008) destacan la importancia de las inversiones realizadas para evitar la movilidad del capital humano en las empresas turísticas, a pesar de que el sector suele considerarlo un gasto y resta importancia a dichas inversiones.

Atendiendo a lo anterior, proponemos que las prácticas de RRHH de retención pueden mejorar los resultados de la organización, particularmente el aumento de los ingresos de explotación de las empresas. Por tanto, planteamos la siguiente hipótesis de investigación:

H4. La adopción de prácticas de RRHH de retención se relaciona positivamente con el incremento de los ingresos de explotación de las empresas.

\section{METODOLOGÍA}

\subsection{Población, recogida de información y muestra}

En este trabajo de investigación la población objeto de estudio está constituida por empresas de alojamiento hotelero que explotan establecimientos de tres o más estrellas en territorio español, con una plantilla igual o superior a 50 empleados. Las empresas con menos de 50 empleados fueron excluidas de la población para eliminar la posibilidad de incluir organizaciones muy pequeñas que no contaran con procedimientos formales de gestión de RRHH (Collins y Smith, 2006; Youndt y Snell, 2004). La importancia del sector turístico en España está ampliamente reconocida. El país ocupa el tercer lugar como destino turístico mundial (OMT, 2017) y el sector aporta un 11,2\% al PIB (INE, 2017a). La capacidad hotelera en España es de más de 1,4 millones de camas las cuales cubren una demanda de más de 330 millones de pernoctaciones anuales (INE, 2017b).

La información necesaria para contrastar las hipótesis planteadas se obtuvo a partir de dos fuentes de información. Por una parte, se hizo uso de la información contenida en una base de datos creada a partir una encuesta realizada, entre septiembre de 2011 y marzo de 
2012, a las empresas objeto de estudio. Esta encuesta se realizó entre las 523 empresas que constituían en aquel momento el total de la población. Los datos recogidos pusieron de manifiesto que la mayoría de los encuestados ejercían funciones de dirección general y ostentaban una antigüedad superior a 10 años en la empresa. El proceso de recogida de datos finalizó con la recepción de un total de 109 cuestionarios válidos. La tabla 1 detalla el número de empresas que conformaron tanto la población como la muestra, distribuidas por Comunidades Autónomas.

Tabla 1

COMPARACIÓN DEL NÚMERO DE EMPRESAS DE LA POBLACIÓN Y DE LA MUESTRA POR COMUNIDADES AUTÓNOMAS

\begin{tabular}{|c|c|c|c|c|c|}
\hline \multirow{2}{*}{$\begin{array}{l}\text { Comunidad } \\
\text { autónoma }\end{array}$} & \multicolumn{2}{|c|}{ Población } & \multicolumn{2}{|c|}{ Muestra } & \multirow[b]{2}{*}{ Diferencia } \\
\hline & \begin{tabular}{c|}
$\mathbf{N}^{\mathbf{0}}$ \\
Empresas
\end{tabular} & Porcentaje & $\begin{array}{c}\mathbf{N}^{\circ} \\
\text { Empresas }\end{array}$ & Porcentaje & \\
\hline Cataluña & 115 & $21,99 \%$ & 17 & $15,60 \%$ & $-6,39 \%$ \\
\hline Islas Baleares & 114 & $21,80 \%$ & 18 & $16,51 \%$ & $-5,28 \%$ \\
\hline Canarias & 65 & $12,43 \%$ & 17 & $15,60 \%$ & $3,17 \%$ \\
\hline Andalucía & 62 & $11,85 \%$ & 11 & $10,09 \%$ & $-1,76 \%$ \\
\hline Comunidad de Madrid & 55 & $10,52 \%$ & 15 & $13,76 \%$ & $3,25 \%$ \\
\hline Comunidad Valenciana & 44 & $8,41 \%$ & 12 & $11,01 \%$ & $2,60 \%$ \\
\hline Castilla y León & 13 & $2,49 \%$ & 4 & $3,67 \%$ & $1,18 \%$ \\
\hline Aragón & 12 & $2,29 \%$ & 2 & $1,83 \%$ & $-0,46 \%$ \\
\hline Galicia & 10 & $1,91 \%$ & 4 & $3,67 \%$ & $1,76 \%$ \\
\hline Principado de Asturias & 6 & $1,15 \%$ & 1 & $0,92 \%$ & $-0,23 \%$ \\
\hline Cantabria & 5 & $0,96 \%$ & 3 & $2,75 \%$ & $1,80 \%$ \\
\hline Comunidad de Navarra & 5 & $0,96 \%$ & 1 & $0,92 \%$ & $-0,04 \%$ \\
\hline País Vasco & 5 & $0,96 \%$ & 1 & $0,92 \%$ & $-0,04 \%$ \\
\hline Región de Murcia & 5 & $0,96 \%$ & 1 & $0,92 \%$ & $-0,04 \%$ \\
\hline Castilla-La Mancha & 3 & $0,57 \%$ & 1 & $0,92 \%$ & $0,34 \%$ \\
\hline Extremadura & 3 & $0,57 \%$ & 1 & $0,92 \%$ & $0,34 \%$ \\
\hline La Rioja & 1 & $0,19 \%$ & 0 & $0,00 \%$ & $-0,19 \%$ \\
\hline TOTAL & 523 & $100,00 \%$ & 109 & $100,00 \%$ & 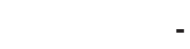 \\
\hline
\end{tabular}

Los ítems incluidos en el cuestionario permitieron medir las variables prácticas de RRHH de reclutamiento-selección, prácticas de RRHH humanos de retención, y capital humano. Asimismo, en el cuestionario se preguntó a los encuestados por el número de empleados de la empresa, dato que se usó como criterio para determinar el tamaño de la misma. 
La otra fuente de información utilizada en este estudio fue la base de datos Sistema de Análisis de Balances Ibéricos (SABI). SABI recoge información financiera contenida en las cuentas anuales, así como otros datos relativos a empresas participadas, actividades, accionistas, etc., de más de 1.250.000 empresas españolas. A partir de esta base de datos se obtuvo la cifra de ingresos de explotación correspondientes a los ejercicios económicos 2011, 2012, 2013, 2014 y 2015 de las empresas estudiadas. Respecto a este punto, cabe señalar que solo se pudo obtener, de forma clara e inequívoca, la información correspondiente a 52 de las 109 empresas que formaban parte de la base de datos anteriormente citada, fundamentalmente por dos razones. En primer lugar, para algunas empresas no existían las cifras de ingresos de explotación correspondientes a todos los años analizados y, por tanto, fueron descartadas. En segundo lugar, algunas empresas recogidas en la primera base de datos ya no figuraban en esta segunda fuente de información o no estaban con el nombre y dirección exactos con los que se habían registrado anteriormente. Con objeto de ser rigurosos se usó solo la información proporcionada por SABI cuando no existían dudas de que se trataba de la misma empresa a la que se había encuestado para obtener la primera base de datos.

\subsection{Escalas de medida}

Las variables prácticas de RRHH de reclutamiento-selección, prácticas de RRHH de retención y capital humano se midieron con una escala tipo Likert de siete puntos, donde 1 significaba "total desacuerdo" y 7 "total acuerdo". Para medir las prácticas de RRHH de reclutamiento-selección y de retención se adaptaron las escalas propuestas en los estudios de Collins (2000), Collins y Smith (2006) y López-Cabrales y Valle-Cabrera (2008). El capital humano se midió usando la escala de Youndt y Snell (2004), que también ha sido utilizada, posteriormente, en los trabajos de Subramaniam y Youndt (2005), y Yamao, de Cieri, y Hutchings (2009). Por su parte, el tamaño de la empresa se midió mediante la transformación a logaritmo natural del número de empleados. Los ítems usados para medir estas variables figuran en las tablas 2,3 y 4.

Como señalamos anteriormente, a partir de la base de datos SABI se obtuvo la cifra de ingresos de explotación de los años 2011 a 2015. Posteriormente, se calculó la tasa de crecimiento de dichos ingresos de explotación en el periodo de un año (2011-2012) desde la realización de la encuesta. De forma similar, se calcularon las tasas de crecimiento que se produjeron en los ingresos de explotación de las empresas durante los dos (2011-2013), tres (2011-2014) y cuatro (2011-2015) años siguientes a la realización de la encuesta. En los anexos 1 y 2 se muestra el crecimiento porcentual de los ingresos de explotación de las empresas que forman parte del estudio, distinguiendo entre empresas medianas (entre 50 y 250 empleados), y empresas grandes (más de 250 empleados). Como puede observarse, la muestra incluye 37 empresas medianas y 15 empresas grandes.

En este estudio se controló estadísticamente el efecto del tamaño de la empresa sobre el capital humano y el crecimiento de los ingresos de explotación. Dado que las empresas más grandes cuentan con ventajas, tales como economías de escala, mayor poder de negociación y más capacidad financiera, investigaciones previas señalan que el tamaño de la empresa puede estar relacionado con los resultados organizativos (Amaraet al., 2008; Avermaeteet al., 2003; Hsu y Sabherwal, 2012). 


\subsection{Análisis de resultados}

En las tablas 2 y 3 se observan los datos del análisis factorial exploratorio realizado sobre las escalas de cuatro ítems usadas para medir las prácticas de RRHH de reclutamiento-selección y las prácticas de RRHH de retención. El análisis proporcionó una solución de un solo factor, en ambas escalas, que explica el $71 \%$ y el $59 \%$ de la varianza total, respectivamente.

Tabla 2

ANÁLISIS DE COMPONENTES PRINCIPALES DE LA ESCALA QUE MIDE LAS PRÁCTICAS DE RRHH DE RECLUTAMIENTO-SELECCIÓN

\begin{tabular}{|l|c|}
\hline \multicolumn{1}{|c|}{ Ítems } & Cargas factoriales \\
\hline $\begin{array}{l}\text { El proceso de selección de candidatos a ocupar puestos de responsabilidad es } \\
\text { intenso (numerosas entrevistas, pruebas, diferentes fuentes de reclutamiento, } \\
\text { etc.) }\end{array}$ &, 709 \\
\hline $\begin{array}{l}\text { Entre las características valoradas en la selección, el potencial del candidato } \\
\text { ostenta un peso importante }\end{array}$ &, 852 \\
\hline $\begin{array}{l}\text { Las habilidades para relacionarse con otras personas son un criterio para la } \\
\text { selección de candidatos }\end{array}$ &, 895 \\
\hline $\begin{array}{l}\text { Las habilidades para colaborar y trabajar en equipo son un criterio para la } \\
\text { selección de candidatos }\end{array}$ &, 892 \\
\hline \multicolumn{2}{|c|}{ Porcentaje de varianza total explicada: $70,65 \%$} \\
\hline \multicolumn{2}{|c|}{ Alfa de Cronbach: 0,85} \\
\hline
\end{tabular}

Tabla 3

ANÁLISIS DE COMPONENTES PRINCIPALES DE LA ESCALA QUE MIDE LAS PRÁCTICAS DE RRHH DE RETENCIÓN

\begin{tabular}{|l|c|}
\hline \multicolumn{1}{|c|}{ Ítems } & Cargas factoriales \\
\hline $\begin{array}{l}\text { En el proceso de selección de candidatos involucramos a los responsables } \\
\text { de departamentos y/o directores de unidades alojativas para asegurarnos que } \\
\text { contratamos a las personas adecuadas }\end{array}$ &, 687 \\
\hline Promovemos eventos sociales para que los empleados se conozcan entre sí &, 763 \\
\hline $\begin{array}{l}\text { La remuneración a directivos y cargos intermedios es superior a la media del } \\
\text { sector }\end{array}$ &, 796 \\
\hline Los empleados son conscientes de sus posibilidades de promoción interna &, 816 \\
\hline Porcentaje de varianza total explicada: $58,87 \%$ & \\
\hline \multicolumn{2}{|c|}{ Alfa de Cronbach: 0,72} \\
\hline
\end{tabular}


La tabla 4 recoge los resultados del análisis de componentes principales con rotación varimax de los cuatro ítems que se usaron para medir el capital humano. El análisis factorial exploratorio generó una solución de un solo factor que explica el 82\% de la varianza total.

\section{Tabla 4 \\ ANÁLISIS DE COMPONENTES PRINCIPALES DE LA ESCALA QUE MIDE EL CAPITAL HUMANO}

\begin{tabular}{|l|c|}
\hline \multicolumn{1}{|c|}{ Ítems } & Cargas factoriales \\
\hline $\begin{array}{l}\text { Nuestros empleados están ampliamente considerados como los } \\
\text { mejores del sector }\end{array}$ &, 876 \\
\hline Nuestros empleados son creativos y brillantes &, 901 \\
\hline $\begin{array}{l}\text { Nuestros empleados son expertos en su puesto de trabajo y funciones } \\
\text { particulares }\end{array}$ &, 933 \\
\hline Nuestros empleados desarrollan nuevas ideas y conocimiento \\
\hline \multicolumn{2}{|c|}{ Porcentaje de varianza total explicada: 81,62\% } \\
\hline \multicolumn{2}{|c|}{ Alfa de Cronbach: 0,92 } \\
\hline
\end{tabular}

A continuación, se exponen los resultados obtenidos en los análisis de regresión realizados sobre el capital humano y sobre el crecimiento porcentual de los ingresos de explotación en los periodos analizados. Como puede observarse, los valores que presenta el nivel de tolerancia en todas las variables oscilan entre 0,921 y 0,971 , lo que indica que no hay problemas de multicolinealidad (Hair et al., 2004). Tal y como se indicó anteriormente, en los modelos se incluyó el tamaño de la empresa como variable de control.

Respecto a la regresión de predicción del capital humano, señalar que las hipótesis H1 y H2 proponen que las prácticas de RRHH de reclutamiento-selección y las prácticas de RRHH de retención se relacionan positivamente con el nivel de capital humano de la empresa. Como se observa en la tabla 5, existe un efecto positivo y significativo tanto de las prácticas de reclutamiento-selección $(\beta=0,315 ; t=4,249, p<0,01)$ como de las prácticas de retención $(\beta=0,550 ; t=6,984, p<0,01)$. Por consiguiente, se aceptan la hipótesis H1 y H2. Asimismo, los análisis evidencian que la variable de control, el tamaño de la empresa, también se relaciona positiva y significativamente con el capital humano. 
Tabla 5

REGRESIÓN DE PREDICCIÓN DEL CAPITAL HUMANO (N=52)

\begin{tabular}{|c|c|c|c|c|c|c|}
\hline Variables independientes & Beta & $t$ & $p$ & tolerancia & $R^{2}$ corregida & Cambio en $F$ \\
\hline $\begin{array}{l}\text { Prácticas de RRHH de } \\
\text { reclutamiento-selección }\end{array}$ &, 315 & 4,249 & 0,000 & ,961 & \multirow{3}{*}{,455 } & \multirow{3}{*}{29,71} \\
\hline $\begin{array}{l}\text { Prácticas de RRHH de } \\
\text { retención }\end{array}$ &, 550 & 6,984 & 0,000 & ,971 & & \\
\hline Tamaño de la empresa & ,210 & 2,787 & 0,006 & ,934 & & \\
\hline
\end{tabular}

En cuanto a la regresión de predicción del crecimiento de los ingresos de explotación, indicar que las hipótesis H3 y H4 plantean que la adopción de prácticas de RRHH de reclutamiento-selección y de retención se relacionan positivamente con el crecimiento de los ingresos de explotación de las empresas. Las tablas 6, 7, 8 y 9 recogen los modelos de regresión del crecimiento porcentual de los ingresos en los periodos 2011-2012, 20112013,2011-2014 y 2011-2015, respectivamente, esto es, se trata de evaluar la evolución de los ingresos de las empresas en los años siguientes a la fecha de realización de la encuesta. Concretamente, se analiza su evolución en el periodo de un año, dos años, tres años y cuatro años desde que las empresas respondieron a la encuesta. Como puede observarse, los resultados reflejan que existe relación estadísticamente significativa entre las prácticas de reclutamiento-selección y el crecimiento de los ingresos de explotación para todos los periodos considerados a excepción del periodo 2011-2015. Indicar que el nivel de significación de esta relación, en el periodo 2011-2013, es de 0,1. Por tanto, se acepta la hipótesis H3. Sin embargo, no existe evidencia para la relación formulada en la hipótesis H4, que proponía una relación positiva entre las prácticas de retención y el incremento de los resultados de explotación. Por otra parte, los datos también muestran una relación positiva y significativa entre el tamaño de la empresa y el crecimiento de los ingresos de explotación en todos los periodos analizados, a excepción del periodo 2011-2012.

\section{Tabla 6 \\ REGRESIÓN DE PREDICCIÓN DEL CRECIMIENTO DE LOS INGRESOS DE EXPLOTACIÓN EN EL PERIODO 2011-2012. (N=52)}

\begin{tabular}{|c|c|c|c|c|c|c|}
\hline Variables independientes & Beta & $t$ & $p$ & tolerancia & $R^{2}$ corregida & Cambio en $F$ \\
\hline $\begin{array}{l}\text { Prácticas de RRHH de } \\
\text { reclutamiento-selección }\end{array}$ & ,381 & 3,022 & ,004 & ,958 & \multirow{3}{*}{, 163} & \multirow{3}{*}{4,583} \\
\hline $\begin{array}{l}\text { Prácticas de RRHH de } \\
\text { retención }\end{array}$ &,- 006 &,- 050 & ,960 & ,960 & & \\
\hline Tamaño de la empresa & , 190 & 1,479 & ,145 & ,923 & & \\
\hline
\end{tabular}


Tabla 7

REGRESIÓN DE PREDICCIÓN DEL CRECIMIENTO DE LOS INGRESOS DE EXPLOTACIÓN EN EL PERIODO 2011-2013. (N=52)

\begin{tabular}{|c|c|c|c|c|c|c|}
\hline $\begin{array}{l}\text { Variables } \\
\text { independientes }\end{array}$ & Beta & $t$ & $p$ & tolerancia & $R^{2}$ corregida & Cambio en $F$ \\
\hline $\begin{array}{l}\text { Prácticas de RRHH de } \\
\text { reclutamiento-selección }\end{array}$ & 231 & 1,891 &, 064 & ,953 & \multirow{3}{*}{,203 } & \multirow{3}{*}{5,748} \\
\hline $\begin{array}{l}\text { Prácticas de RRHH de } \\
\text { retención }\end{array}$ & ,046 &, 380 & ,705 & ,962 & & \\
\hline Tamaño de la empresa & ,384 & 3,089 & 003 & ,922 & & \\
\hline
\end{tabular}

Tabla 8

REGRESIÓN DE PREDICCIÓN DEL CRECIMIENTO DE LOS INGRESOS DE EXPLOTACIÓN EN EL PERIODO 2011-2014. (N=52)

\begin{tabular}{|c|c|c|c|c|c|c|}
\hline $\begin{array}{l}\text { Variables } \\
\text { independientes }\end{array}$ & Beta & $t$ & $p$ & tolerancia & $R^{2}$ corregida & Cambio en $F$ \\
\hline $\begin{array}{l}\text { Prácticas de RRHH de } \\
\text { reclutamiento-selección }\end{array}$ & ,261 & 2,148 & ,036 & ,953 & \multirow{3}{*}{,223 } & \multirow{3}{*}{6,274} \\
\hline $\begin{array}{l}\text { Prácticas de RRHH de } \\
\text { retención }\end{array}$ &, 052 &, 430 & ,669 & ,964 & & \\
\hline Tamaño de la empresa &, 382 & 3,090 & ,003 & ,922 & & \\
\hline
\end{tabular}

Tabla 9

REGRESIÓN DE PREDICCIÓN DEL CRECIMIENTO DE LOS INGRESOS DE EXPLOTACIÓN EN EL PERIODO 2011-2015. (N=52)

\begin{tabular}{|c|c|c|c|c|c|c|}
\hline $\begin{array}{l}\text { Variables } \\
\text { independientes }\end{array}$ & Beta & $t$ & $p$ & tolerancia & $R^{2}$ corregida & Cambio en $F$ \\
\hline $\begin{array}{l}\text { Prácticas de RRHH de } \\
\text { reclutamiento-selección }\end{array}$ &, 131 & 1,026 &, 310 & ,957 & \multirow{3}{*}{, 207} & \multirow{3}{*}{5,437} \\
\hline $\begin{array}{l}\text { Prácticas de RRHH de } \\
\text { retención }\end{array}$ &,- 002 &,- 015 & ,988 & ,956 & & \\
\hline Tamaño de la empresa &, 462 & 3,556 & 001 & ,921 & & \\
\hline
\end{tabular}




\section{CONCLUSIONES E IMPLICACIONES}

El objetivo de esta investigación es evaluar el impacto de las prácticas de RRHH de reclutamiento-selección y de retención sobre el capital humano y el crecimiento de los ingresos de explotación en empresas hoteleras situadas en todo el territorio español. La importancia de la investigación radica en la escasez de estudios similares en el campo de la hostelería y turismo. El turismo en España es uno de los pilares de la economía del país y los empleados del sector constituyen un elemento crítico para el éxito de las empresas.

Los resultados sugieren que la aplicación tanto de prácticas exhaustivas de RRHH en reclutamiento y selección como de políticas de retención de los empleados favorece el desarrollo de capital humano. En concreto, unas prácticas rigurosas de reclutamiento y selección permiten atraer personal cualificado a las empresas que mejora los niveles de conocimiento, habilidades y destrezas de sus miembros. De igual modo, las prácticas de retención, al incentivar que los empleados no abandonen la organización, favorecen que los mismos desarrollen conocimientos específicos que, también, contribuye a mejorar el nivel de capital humano. Investigaciones previas ponen de manifiesto discrepancias en los estudios que analizan la relación entre prácticas de reclutamiento-selección y capital humano. Los resultados alcanzados en este estudio pueden asimilarse a los obtenidos por López-Cabrales y Valle (2008). No obstante, usando una escala más centrada en prácticas de reclutamiento, el trabajo de Youndt y Snell (2004) encuentra una relación positiva con el capital humano, mientras que Yamao et al. (2009) no encuentran tal relación significativa. La investigación de Cabello et al. (2011), tampoco muestra relación entre ciertas prácticas de selección y el valor y especificidad del capital humano. Las razones de estos resultados contradictorios es posible que se deriven de diferencias entre los tipos y el tamaño de las empresas que constituyen la muestra en las distintas investigaciones, que pueden influir en los métodos específicos utilizados por las empresas para reclutar y seleccionar al personal.

Por otra parte, los datos indican que las empresas que utilizaron prácticas exhaustivas de reclutamiento-selección obtuvieron mayores incrementos en sus ingresos de explotación hasta los tres años siguientes a la realización de la encuesta. Sin embargo, no existe evidencia de tal relación positiva para el crecimiento de dichos ingresos en un periodo que abarca los siguientes cuatro años desde la realización de la encuesta. Una posible explicación a la falta de relación a partir del tercer año desde que se obtuvieron los datos puede encontrarse en el hecho de que las empresas dejaran de utilizar este tipo de prácticas, o que la influencia de éstas puede verse disminuida con el tiempo. Asimismo, aunque poco probable, podría ser posible que, con el paso del tiempo, se produjera una modificación sustancial de la dimensión de las empresas que provocara la reducción de sus ingresos de explotación, pero no de sus resultados de explotación. Los resultados encontrados en este estudio apoyan los argumentos teóricos de Saddam y Mansor (2015) y pueden asimilarse a los obtenidos en los trabajos empíricos de Mustapha et al. (2013) y Saifalislam et al. (2014), para quienes los procesos de reclutamiento y selección contribuyen al desempeño organizativo.

Contrariamente a lo esperado, los datos no muestran una relación positiva entre las prácticas de RRHH de retención y el crecimiento de los ingresos de explotación y, en tal sentido, no apoyan los planteamientos teóricos de Minbaeva et al. (2009). Este hallazgo 
puede deberse a que, tratándose del sector servicios, la acumulación de experiencia en una misma empresa es posible que no sea tan crítica como en otros sectores. En este ámbito, es relativamente sencillo contratar personal con experiencia en puestos similares en otras empresas del sector, sin necesidad de que el nuevo empleado requiera un tiempo de permanencia en la empresa para realizar sus tareas de manera efectiva. Otra posible explicación puede deberse a la inercia que puede generarse en los empleados que permanecen durante tiempo en la organización, en tanto que los nuevos podrían mostrar mayor interés en lograr mejorar los datos financieros de sus empresas. Asimismo, es posible que el tamaño de la muestra no permita que se pongan de manifiesto relaciones significativas entre ambas variables.

Por otra parte, los resultados muestran que el tamaño de la empresa influye positivamente tanto en el nivel de capital humano como en la consecución de mayores incrementos en los ingresos de explotación. En línea con investigaciones previas, parece lógico pensar que las empresas de mayor dimensión pueden contratar a los candidatos más valiosos y, además, cuentan con más recursos a su disposición para mejorar sus resultados financieros.

Esta ampliamente reconocida la importancia de aplicar políticas rigurosas que permitan reclutar y seleccionar a los mejores candidatos del mercado. Asimismo, se considera que la fidelidad y el compromiso de los trabajadores con su propia empresa es un factor importante en un mercado laboral cada vez más agresivo. Este estudio encuentra que ambas políticas permiten mejorar el capital humano de las empresas de alojamiento turístico. Por consiguiente, los directivos de estas empresas deben favorecer la aplicación de prácticas de reclutamiento, selección y retención para disponer de capital humano valioso, que asuma como propios los objetivos empresariales, que se sienta justamente valorado en el desempeño de sus funciones, y que disponga de medios para su desarrollo personal y profesional. No obstante, cuando se trata de lograr mejores resultados financieros, los resultados del estudio sugieren que los directivos deben prestar especial atención a las prácticas de reclutamiento y selección.

Finalmente, las empresas del sector hotelero interesadas en mejorar su desempeño necesitan centrar sus esfuerzos en disponer de la mejor fuerza laboral ya que ésta constituye un elemento clave en la valoración del producto que recibe el cliente lo que representa un aspecto esencial para lograr la fidelización del mismo. Por ello, es vital la utilización de prácticas exhaustivas de RRHH en reclutamiento y selección que permita atraer talento a las empresas. Las características propias del sector, donde predominan los puestos de baja cualificación parece que hacen más interesante la inversión en reclutar personal cualificado que en retenerlo, lo que sugiere que la rotación de personal es relativamente más sencilla y rápida de ser recuperada mediante adecuadas prácticas de reclutamiento y selección.

En la evaluación de las aportaciones de este estudio se deben tener en cuenta algunas limitaciones, las cuales pueden constituir posibilidades de investigación futura. Una limitación surge porque las prácticas de RRHH se midieron a partir de la percepción de un solo informante por empresa, normalmente el director, y no se obtuvo la opinión de los empleados. Asimismo, futuras investigaciones podrían incorporar otras prácticas de RRHH no incluidas en este estudio, tales como formación, participación o empoderamiento, las cuales pueden proporcionar un mayor poder explicativo a la relación entre 
prácticas de gestión de RRHH y desempeño organizativo. Adicionalmente, los resultados deben ser analizados teniendo en consideración factores del entorno económico. La presente investigación se enmarca en una época de crisis sufrida por la economía española, a partir del año 2008,y posterior recuperación, en el año 2014. Esta situación de cambios en el ciclo económico podría moderar de algún modo las relaciones analizadas.

\section{BIBLIOGRAFÍA}

ALBRECHT, S.L., BAKKER, A.B., GRUMAN, J.A., MACEY, W.H. y SAKS, A.M. (2015): «Employee engagement, human resource management practices and competitive advantage: An integrated approach», Journal of Organizational Effectiveness: People and Performance, vol. 2 (1), pp. 7-35.

ALLEN, M.R., ERICKSEN, J. y COLLINS, C.J. (2013): «Human resource management, employee exchange relationships, and performance in small businesses», Human Resource Management, vol. 52 (2), pp. 153-174.

AMARA, N., LANDRY, R., BECHEIKHB, N. y OUIMET, M. (2008): «Learning and novelty of innovation in established manufacturing SMEs», Technovation, vol. 28 (8), pp. 450-463.

AVERMAETE, T., VIAENE, J., MORGAN, E.J. y CRAWFORD, N. (2003): «Determinants of innovation in small food firms», European Journal of Innovation Management, vol. 6 (1), pp. 8-17.

BECKER, B.E. y HUSELID, M.A. (1998): «High performance work systems and firm performance: A synthesis of research and managerial implications», Research in Personnel and Human Resource Management, vol. 16, pp. 53-101.

BECKER, G.S. (2002): The age of human capital, pp. 3-8, 15 de junio de 2018.Disponible en:http://media.hoover.org/sites/default/files/documents/0817928928_3.pdf.

CABELLO, C., LÓPEZ-CABRALES, A. Y VALLE, R. (2011): "Leveraging the innovative performance of human capital through HRM and social capital in Spanish firms", International Journal of Human Resource Management, vol. 22 (4), pp. 807-828.

CHAN, S. H. y KUOK, O.M. (2011): «A Study of Human Resources Recruitment, Selection, and Retention Issues in the Hospitality and Tourism Industry in Macau»,Journal of Human Resources in Hospitality y Tourism, vol. 10 (4), pp. 421-441.

COLLINS, C.J. (2000): Strategic human resources management and knowledge creation capability: Examining the black-box between HR and firm performance». Tesis doctoral, University of Maryland, College Park, MD. 898-925.

COLLINS, C.J. y SMITH, K.G. (2006):«Knowledge exchange and combination: the role of human resource practices in the performance of high-technology firms», Academy of Management Journal, vol. 49 (3), pp. 544-560.

DELERY, J.E. y ROUMPI, D. (2017): «Strategic human resource management, human capital and competitive advantage: is the field going in circles?», Human Resource Management Journal, vol. 27 (1), pp. 1-21.

DELERY, J.E. y SHAW, J.D. (2001): «The strategic management of people in work organizations: Review, synthesis, and extension», en Research in Personnel and Human 
Resources Management, vol. 20, Emerald Group Publishing Limited, pp.165-197, 3 de agosto de 2018. Disponible en: https://www.emeraldinsight.com/doi/abs/10.1016/S0742-7301\%2801\%2920003-6

DÍAZ-FERNÁNDEZ, M., LÓPEZ-CABRALES, A. y VALLE-CABRERA, R. (2014): «A contingent approach to the role of human capital and competencies on firm strategy», BRQ Business Research Quarterly, vol. 17 (3), pp. 205-222.

FRENDE-VEGA, M. A., BIEDMA-FERRER, J. M. y ARANA-JIMÉNEZ, M. (2017): «Influencia de la percepción y metodologías docentes aplicadas en el rendimiento académico de los estudiantes de la asignatura de dirección de recursos humanos en las empresas turísticas», Cuadernos de Turismo, no 39, pp. 149-166.

GUTIÉRREZ-BRONCANO, S. y RUBIO-ANDRÉS. M. (2009): «El factor humano en los sistemas de gestión de calidad del servicio: un cambio de cultura en las empresas turísticas», Cuadernos de Turismo, n 23, pp. 129-147.

HAIR, J., ANDERSON, R., TATHAM, R. y BLACK, W. (2004): Análisis multivariante (5a ed.). Madrid, Pearson, Prentice Hall.

HAMILTON, R. y DAVISON, H.K. (2018): «The search for skills: Knowledge stars and innovation in the hiring process», Business Horizons, vol. 61 (3), pp. 409-419.

HARKY, Y.F.M. (2018): «The Significance of Recruitment and Selection on Organizational Performance: The Case of Private owned Organizations in Erbil, North of Iraq», International Journal of Contemporary Research and Review, vol. 9 (2). https://doi. org/https://doi.org/10.15520/ijcrr/2018/9/02/422.

HJALAGER, A.M. (2002): «Repairing innovation defectiveness in tourism»,Tourism management, vol. 23, $\mathrm{n}^{\circ} 5$, pp. 465-474.

HSU, I. C. y SABHERWAL, R. (2012): «Relationship between intellectual capital and knowledge management: An empirical investigation»,Decision Science, Vol. 43, n 3, pp. 489-524.

INE. Instituto Nacional de Estadística, (2017a): Cuenta Satélite del Turismo de España. Base 2010 Serie 2010-2016. 1 de junio de 2018. Disponible en: http://www.ine.es/ prensa/cst_2016.pdf.

INE. Instituto Nacional de Estadística (2017b): Datos de pernoctaciones año 2016. 1 de junio de 2018. Disponible en: http://www.ine.es/dynt3/inebase/es/index. htm? padre $=238 \& \mathrm{dh}=1$.

KAUFMAN, B. E. (2012): «Strategic Human Resource Management Research in the United States: A Failing Grade After 30 Years?», Academy of Management Perspectives, vol. 26 (2), pp. 12-36.

KIM, T., KIM, W.G., PARK, S.S-S., LEE, G. y JEE, B. (2012): «Intellectual Capital and Business Performance: What Structural Relationships Do They Have in Upper-Upscale Hotels?»,International Journal of Tourism Research, vol. 14, (4), pp, 391-408.

KUSLUVAN, S., KUSLUVAN, Z., ILHAN, H. y BUYRUK, L. (2010): «A Review of Human Resources Management Issues in the Tourism and Hospitality Industry», Cornell Hospitality Quarterly, vol. 51 (2), pp. 171-214.

LADO, A.A. y WILSON, M.C. (1994): «Human Resource Systems and Sustained Competitive Advantage: A Competency-Based Perspective», The Academy of Management Review, vol. 19 (4), pp. 699-727. 
LAM, T., LO, A. y CHAN, J. (2002): «New employees' turnover intentions and organizational commitment in the Hong Kong hotel industry», Journal of Hospitality \& Tourism Research, vol. 26 (3), pp. 217-234.

LEANA, C. y VAN BUREN, H. (1999): «Organizational social capital and employment relations». Academy of Management Review, vol. 24 (3), pp. 538-555.

LEPISTÖ, L. e IHANTOLA, E.M. (2018): «Understanding the recruitment and selection processes of management accountants: An explorative study», Qualitative Research in Accounting y Management, vol. 15 (1), pp. 104-123.

LILLO-BAÑULS, A. (2009): «El papel del capital humano en el sector turístico: algunas reflexiones y propuestas», Cuadernos de Turismo, no 24, pp. 53-64.

LILLO-BAÑULS, A., RAMÓN-RODRÍGUEZ, A.B. y SEVILLA-JIMÉNEZ. M. (2007), «El capital humano como factor estratégico para la competitividad del sector turístico», Cuadernos de Turismo, no 19 , pp. 47-69.

LÓPEZ-CABRALES, A. y VALLE, R. (2008): "Capital humano, prácticas de gestión empresarial: ¿Están relacionadas?”, Revista Europea de Dirección y Economía de la Empresa, vol. 17 (2), pp. 155-178.

MADERA, J.M., DAWSON, M., GUCHAIT, P. y BELARMINO, A.M. (2017): «Strategic human resources management research in hospitality and tourism: A review of current literature and suggestions for the future», International Journal of Contemporary Hospitality Management, vol. 29 (1), pp. 48-67.

MAHMOOD, M. (2015): «Strategy, structure, and HRM policy orientation: Employee recruitment and selection practices in multinational subsidiaries», Asia Pacific Journal of Human Resources, vol. 53 (3), pp. 331-350.

MARKOS, S. y SRIDEVI, M.S. (2010): «Employee Engagement: The Key to Improving Performance», International Journal of Business and Management, vol. 5 (12), pp. 89-96.

MINBAEVA, D., FOSS, N. y SNELL, S. (2009): «Guest editors' introduction: Bringing the knowledge perspective into HRM», Human Resource Management, vol. 48 (4), pp. 477-483.

MUSTAPHA, A.M., ILESANMI, O.A. y AREMU, M. (2013): «The impact of wellplanned recruitment and selection process on corporate performance in Nigerian banking industry: A case study of First Bank PLC 2004-2011», International Journal of Academic Research in Business and Social Sciences, vol. 3 (9), pp. 633-648.

NAVEEN, S. (2013): «A Study On Recruitment y Selection Process With Reference To Three Industries, Cement Industry, Electronics Industry, Sugar Industry In Krishna Dt Ap,India», Journal of Business and Management, vol. 15 (5), pp. 60-67.

OMT. Organización Mundial del Turismo (2017): Panorama OMT del Turismo Internacional, Edición 2017, 15 de junio de 2018. Disponible en: https://www.e-unwto.org/doi/ pdf/10.18111/9789284419043.

PASBAN, M. y NOJEDEH, S.H. (2016): «A Review of the Role of Human Capital in the Organization», Procedia-Social and Behavioral Sciences, vol. 230, pp. 249-253.

PETTS, N. (1997): «Building growth on core competences - a practical approach», Long Range Planning, vol. 30 (4), pp. 551-561. 
ROBINSON D., PERRYMAN S. y HAYDAY S. (2004): The Drivers of Employee Engagement Report 408, Institute for Employment Studies, UK. Recuperado de: https://www.employment-studies.co.uk/system/files/resources/files/408.pdf (acceso 2018, 05).

SADDAM, A.K. y MANSOR, N.N.A. (2015): «The Role of Recruitment and Selection Practices in the Organizational Performance of Iraqi Oil and Gas Sector: A Brief Literature Review», Review of European Studies, vol. 7 (11), p. 348.

SAIFALISLAM, K.M., OSMAN, A. y ALQUDAH, M.K. (2014): «Human Resource Management Practices: Influence of recruitment and selection, and training and development on the organizational performance of the Jordanian Public University», Journal of Business and Management, vol. 16 (5), pp. 43-46.

SHUCK, B. y WOLLARD, K. (2010): «Employee engagement and HRD: A seminal review of the foundations», Human Resource Development Review, vol. 9 (1), pp. 89-110.

STEFFY, B.D. y MAURER, S.D. (1988): «Conceptualizing and Measuring The Economic Effectiveness of Human Resource Activities», The Academy of Management Review, vol. 13 (2), pp. 271-286.

SUBRAMANIAM, M. y YOUNDT, M.A. (2005): «The influence of intellectual capital on the types of innovative capabilities», Academy of Management Journal, vol. 48 (3), pp. 450-463.

WALSH, K., ENZ, C. y CANINA, L. (2008): «The impact of strategic orientation on the intellectual capital investments in customer service firms», Journal of Services Research, vol. 10 (4), pp. 300-317.

WRIGHT, P., DUNFORD, B. y SNELL, S. (2001): «Human resources and the resourcebased view of the firm», Journal of Management, vol. 27 (6), pp 701-721.

WRIGHT, P.M., MCMAHAN, G.C. y MCWILLIAMS, A. (1994): «Human resources and sustained competitive advantage: a resource-based perspective», The International Journal of Human Resource Management, vol. 5 (2), pp. 301-326.

YAMAO, S., DE CIERI, H. y HUTCHINGS, K. (2009): «Transferring subsidiary knowledge to global headquarters: Subsidiary senior executives' perceptions of the role of HR configurations in the development of knowledge stocks», Human Resource Management, vol. 48 (4), pp. 531- 554.

YANG, J.T. y WAN, C.S. (2004): «Advancing organizational effectiveness and knowledge management implementation», Tourism Management, vol. 25 (5), pp. 593-601.

YOUNDT, M.A. y SNELL, S.A. (2004): «Human resource configurations, intellectual capital and organizational performance», Journal of Managerial Issues, vol. XVI (3), pp. 337-360.

ZHONG, L., WAYNE, S.J. y LIDEN, R.C. (2016): «Job engagement, perceived organizational support, high-performance human resource practices, and cultural value orientations: A cross-level investigation: job engagement, pos, and HR practices», Journal of Organizational Behavior, vol. 37 (6), pp. 823-844. 
IMPACTO DE LAS PRÁCTICAS DE RECURSOS HUMANOS EN EL CAPITAL HUMANO Y LOS... 331

\section{Anexo 1}

CRECIMIENTO PORCENTUAL DE LOS INGRESOS EXPLOTACIÓN DE LAS EMPRESAS MEDIANAS

\begin{tabular}{|c|c|c|c|c|}
\hline \multirow{2}{*}{ Empresa } & \multicolumn{4}{|c|}{ Periodo } \\
\hline & 2011-2012 & 2011-2013 & 2011-2014 & 2011-2015 \\
\hline 1 & $-41,08$ & $-65,14$ & $-57,61$ & $-48,94$ \\
\hline 2 & 2,19 & 7,56 & 2,98 & 6,33 \\
\hline 3 & 2,25 & $-1,33$ & $-23,76$ & $-21,86$ \\
\hline 4 & $-20,99$ & 1,32 & 11,38 & 37,67 \\
\hline 5 & $-0,08$ & $-4,75$ & 11,50 & 31,54 \\
\hline 6 & $-15,86$ & $-9,53$ & $\begin{array}{l}-13,99 \\
\end{array}$ & $-19,17$ \\
\hline 7 & 13,75 & 17,76 & 21,85 & 34,49 \\
\hline 8 & $-3,14$ & $-5,13$ & 4,28 & 13,67 \\
\hline 9 & $-28,28$ & $-25,36$ & $-30,15$ & $-30,09$ \\
\hline 10 & $-0,53$ & $-6,46$ & $-9,28$ & $-3,12$ \\
\hline 11 & 3,11 & 10,78 & 20,48 & 23,09 \\
\hline 12 & 10,22 & 11,13 & $-5,35$ & 0,39 \\
\hline 13 & $-5,48$ & $-12,26$ & $-12,26$ & $-2,50$ \\
\hline 14 & $-8,70$ & $-14,62$ & $-12,18$ & $-0,93$ \\
\hline 15 & 2,71 & 3,03 & 7,44 & 12,53 \\
\hline 16 & $-0,28$ & $-14,99$ & $-27,35$ & $-26,02$ \\
\hline 17 & $-0,62$ & $-2,97$ & 2,73 & 13,33 \\
\hline 18 & 7,66 & 9,05 & 14,10 & 20,91 \\
\hline 19 & 1,44 & 7,56 & 25,35 & 37,05 \\
\hline 20 & $-7,83$ & 0,00 & 19,75 & 35,50 \\
\hline 21 & $-11,24$ & $-7,85$ & $-5,84$ & 0,49 \\
\hline 22 & $-31,38$ & $-24,87$ & $-7,34$ & $-16,64$ \\
\hline 23 & $-8,87$ & $-2,59$ & $-2,40$ & $-5,48$ \\
\hline 24 & $-9,26$ & $-17,96$ & $-14,93$ & $-5,05$ \\
\hline 25 & $-5,88$ & $-3,68$ & $-2,44$ & 7,40 \\
\hline 26 & 21,23 & 19,54 & 14,85 & 29,36 \\
\hline 27 & $-16,06$ & $-28,47$ & $-38,61$ & $-5,59$ \\
\hline 28 & 17,57 & 42,24 & 100,12 & 82,31 \\
\hline 29 & $-16,31$ & $-11,35$ & $-32,93$ & 3,96 \\
\hline 30 & 1,65 & 5,49 & 14,85 & 28,38 \\
\hline 31 & $-0,88$ & 9,34 & 15,94 & 15,28 \\
\hline 32 & 5,54 & 6,50 & 31,08 & 44,57 \\
\hline
\end{tabular}




\begin{tabular}{|c|r|r|r|r|}
\hline \multirow{2}{*}{ Empresa } & \multicolumn{4}{|c|}{ Periodo } \\
\cline { 2 - 5 } & $\mathbf{2 0 1 1 - 2 0 1 2}$ & $\mathbf{2 0 1 1 - 2 0 1 3}$ & $\mathbf{2 0 1 1 - 2 0 1 4}$ & $\mathbf{2 0 1 1 - 2 0 1 5}$ \\
\hline 33 & 23,42 & 24,82 & 35,26 & 44,74 \\
\hline 34 & 0,58 & 4,41 & 2,56 & $-1,18$ \\
\hline 35 & $-4,93$ & $-10,89$ & $-0,97$ & $-3,02$ \\
\hline 36 & 10,06 & 13,13 & 13,12 & 36,60 \\
\hline 37 & $-7,10$ & $-6,63$ & $-8,02$ & 27,48 \\
\hline
\end{tabular}

Anexo 2

CRECIMIENTO PORCENTUAL DE LOS INGRESOS EXPLOTACIÓN DE LAS EMPRESAS GRANDES

\begin{tabular}{|c|c|c|c|c|}
\hline \multirow{2}{*}{ Empresa } & \multicolumn{4}{|c|}{ Periodo } \\
\hline & 2011-2012 & 2011-2013 & 2011-2014 & 2011-2015 \\
\hline 1 & 1,35 & 11,82 & 15,85 & 24,80 \\
\hline 2 & $-6,88$ & $-28,27$ & $-24,13$ & $-14,32$ \\
\hline 3 & $-1,19$ & 2,08 & 8,58 & 16,10 \\
\hline 4 & 4,20 & 9,93 & 26,31 & 35,13 \\
\hline 5 & 4,24 & $-0,49$ & $-1,09$ & 4,23 \\
\hline 6 & $-4,66$ & $-6,49$ & $-1,23$ & $-17,96$ \\
\hline 7 & 0,48 & 7,28 & 46,39 & 64,20 \\
\hline 8 & 6,42 & 72,33 & 124,06 & 161,28 \\
\hline 9 & 5,90 & 15,09 & 29,61 & 50,77 \\
\hline 10 & $-4,84$ & $-7,74$ & 4,51 & 38,14 \\
\hline 11 & $-2,33$ & 4,65 & 9,30 & 34,88 \\
\hline 12 & $-9,67$ & $-12,68$ & $-6,26$ & 2,74 \\
\hline 13 & 11,81 & 33,33 & 53,28 & 62,82 \\
\hline 14 & $-0,52$ & 118,14 & 145,83 & 190,68 \\
\hline 15 & $-9,11$ & $-12,51$ & $-4,49$ & 6,96 \\
\hline
\end{tabular}

